\title{
GLOBULAR CLUSTERS IN ELLIPTICAL GALAXIES: CONSTRAINTS ON MERGERS
}

\author{
DUNCAN A. FORBES
}

School of Physics and Astronomy, University of Birmingham, Birmingham, B15 2TT, UK

\section{Introduction}

There exists a relationship between globular cluster mean metallicity and parent galaxy luminosity (e.g. Brodie \& Huchra 1991; Forbes et al. 1996), which appears to be similar to that between stellar metallicity and galaxy luminosity. The globular cluster relation has a similar slope but is offset by about 0.5 dex to lower metallicity. The similarity of these relations suggests that both the globular cluster system and their parent galaxy have shared a common chemical enrichment history. If we can understand the formation and evolution of the globulars, we will also learn something about galaxy formation. With this aim in mind we have created the SAGES (Study of the Astrophysics of Globular clusters in Extragalactic Systems) project. Project members include Brodie, Elson, Forbes, Freeman, Grillmair, Huchra, Kissler-Patig and Schroder. We are using HST Imaging and Keck spectroscopy to study extragalactic globular cluster systems. Further details are given at http://www.ucolick.org/ ${ }^{\sim}$ mkissler/Sages/sages.html.

\section{Results and Discussion}

van den Bergh (1975) has argued that ellipticals have too many globular clusters per unit starlight (called specific frequency, $S_{\mathrm{N}}$ ) to be due to the simple merger of spirals. Spiral galaxies have $S_{\mathrm{N}} \sim 0.5$. This is increased to $S_{\mathrm{N}} \sim 2$ if we take into account the different mass-to-light ratios of spirals relative to ellipticals. A typical elliptical galaxy, with $M_{\mathrm{V}} \sim-21$, has $S_{\mathrm{N}}$ $\sim 4$. Hence there is a factor of two difference. This discrepancy gets larger for more luminous ellipticals. However, it has been suggested that new globular clusters can form in the gas associated with the merger event (Schweizer 1987; Ashman \& Zepf 1992). Recently proto-globular cluster 
candidates have been found in a number of merging systems, largely due to the resolving power of HST. A literature summary is given in Table 1.

TABLE 1. Proto-Globular Clusters

\begin{tabular}{lll}
\hline Galaxy & Merger Type & \% Increase \\
\hline NGC $4038 / 9$ & $\mathrm{~S}+\mathrm{S}$ & $\sim 100$ \\
NGC 7252 & $\mathrm{Sc}+\mathrm{Sc}$ & $\sim 70$ \\
NGC 3610 & $\mathrm{~S}+\mathrm{S}$ & $\sim 70$ \\
NGC 3256 & $\mathrm{~S}+\mathrm{S}$ & $\sim 100$ \\
NGC 3921 & $\mathrm{Sc}+\mathrm{S} 0$ & $\sim 40$ \\
NGC 5128 & $\mathrm{E}+\mathrm{S}$ & $\sim 20$ \\
NGC 5018 & $\mathrm{E}+\mathrm{S}$ & $\sim 10$ \\
NGC 1316 & $\mathrm{E}+\mathrm{S}$ & $0-30$ \\
NGC 1275 & $\mathrm{E}+\mathrm{S}$ & $\leq 10$ \\
\hline
\end{tabular}

From Table 1 it appears that the number of newly created protoglobulars varies with the progenitor types (i.e. the gas content) and that the percentage increase in the total cluster population is $100 \%$ or less. This is in contrast to the discussion above which requires $100 \%$ or more new globular clusters to counter van den Bergh's objection. A further issue to consider, is whether these cluster candidates will ever resemble globular clusters as we known them - there is some circumstantial evidence that they may not (Brodie et al. 1997).

Although the initial evidence was weak, there are now a handful of convincing cases for bimodal globular cluster color (metallicity) distributions in ellipticals (see Forbes, Brodie \& Grillmair 1997). This indicates that some ellipticals have more that one globular cluster population. The observed metallicity distributions rule out a simple monolithic collapse and provide a strong constraint for any globular cluster formation model.

Bimodality has been taken as support for the merger picture of Ashman \& Zepf (1992). Their model would predict the ratio of metal-rich to metalpoor $\left(N_{\mathrm{R}} / N_{\mathrm{P}}\right)$ globulars to be about 1 for an elliptical with $S_{\mathrm{N}} \sim 4$ and about 4 for $S_{\mathrm{N}} \sim 16$. Furthermore the metal-poor peak should have a metallicity around $[\mathrm{Fe} / \mathrm{H}] \sim-1.5$, i.e. to match that of a typical spiral. Some examples of galaxies that do not fit this picture include: NGC 5846, although the ratio $N_{\mathrm{R}} / N_{\mathrm{P}} \sim 3, S_{\mathrm{N}}$ is not high but rather low at 2.8 (Forbes, Brodie \& Huchra 1997). In the case of NGC 4472, the metal-poor peak contains a total of about 4000 globulars (Geisler et al. 1996). This would require about $10-20 L^{*}$ spirals. At the other extreme is NGC 3311 (Secker 


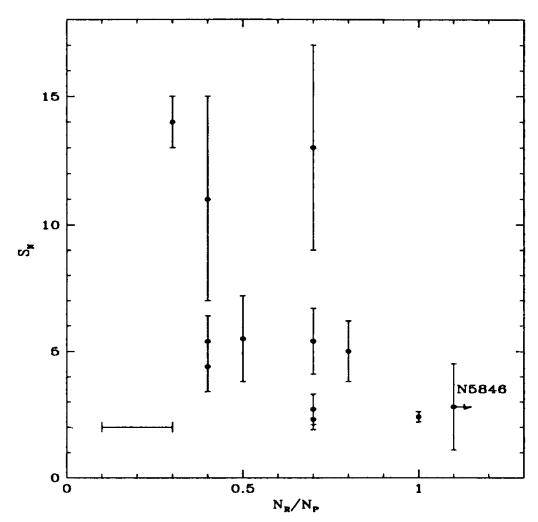

Figure 1. The specific frequency $\left(S_{\mathrm{N}}\right)$ versus the ratio of metal-rich to metal-poor globular clusters. The merger picture predicts a trend of increasing $S_{\mathrm{N}}$ with increasing $N_{\mathrm{R}} / N_{\mathrm{P}}$ ratio, i.e. opposite to that seen.

et al. 1995) which has virtually no globulars with $[\mathrm{Fe} / \mathrm{H}]=-1.5$, i.e. none from $L^{*}$ type spirals.

In Fig. 1 we show $S_{\mathrm{N}}$ against $N_{\mathrm{R}} / N_{\mathrm{P}}$ for 12 large ellipticals (4 of which are $\mathrm{cD}$ galaxies). This figure includes two galaxies from the poster of Geisler $\&$ Lee (this conference). If the overabundant globular cluster systems (i.e. those with high $S_{N}$ values) are due to the creation of more (metal-rich) globular clusters in a merger event, then we would expect a trend of increasing $S_{\mathrm{N}}$ with increasing $N_{\mathrm{R}} / N_{\mathrm{P}}$ ratio. Fig. 1 shows the opposite trend, so that high $S_{\mathrm{N}}$ galaxies actually have a larger fraction of metal-poor globulars.

So although large ellipticals have bimodal metallicity distributions, when examined in detail they do not fit the expectations of the merger picture. What is the situation for low luminosity ellipticals? These are much harder to study given that such galaxies have fewer globular clusters and so any bimodality will be more difficult to detect. Kissler-Patig, Forbes \& Minitti (1997) have combined HST and ground-based imaging of NGC 1427 to obtain what is probably the best photometrically-studied globular cluster system in a low luminosity elliptical. Its color distribution is shown in Fig. 2, indicating that it is unimodal. If NGC 1427 formed by the gaseous merger of two disk galaxies we would expect a bimodal distribution and yet it is clearly unimodal. Unless there is an age-metallicity conspiracy that hides the two globular cluster populations we are forced to conclude that NGC 1427 has only one population and does not fit the merger picture. 


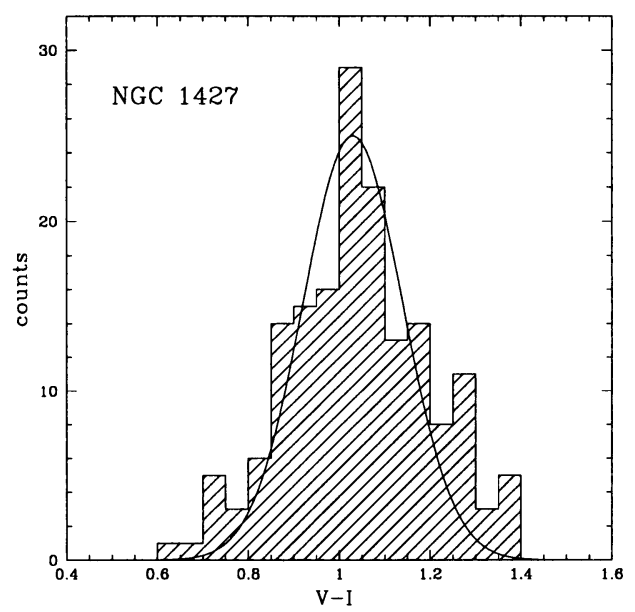

Figure 2. Globular cluster color distribution for NGC $1427\left(M_{\mathrm{V}}=-20.5, S_{\mathrm{N}}=5\right)$. The Gaussian shows the broadening due to photometric errors. The color distribution is consistent with a single, unimodal metallicity.

\section{Concluding Remarks}

It is clear that spiral+spiral mergers are occurring today at redshift $z=0$, and that such gaseous mergers may be forming proto-globular clusters; however they may not be forming in sufficient quantities to resemble a 'normal' large elliptical. Gaseous mergers at $z \geq 1$ predict bimodal metallicity distributions but these are not seen in small ellipticals, and the details do not match the predictions for the large ellipticals. The presence of bimodal distributions rules out a monolithic collapse, so perhaps we should re-consider a two phase collapse (see Forbes, Brodie \& Grillmair 1997).

\section{References}

Ashman K.M., \& Zepf S.E. 1992, ApJ 384, 50

Brodie, J. P., \& Huchra, J. 1991, ApJ, 379, 157

Brodie, J. P., et al. 1997, AJ, submitted

Forbes, D. A., et al. 1996, ApJ, 467, 126

Forbes D.A., Brodie J.P., \& Grillmair, C.J. 1997, AJ 113, 1652

Geisler, D., Lee, M. G., \& Kim, E. 1996, AJ, 111, 1529

Kissler-Patig, M., Forbes, D. A., \& Minitti, D. 1997, MNRAS, submitted

Schweizer, F. 1987, Nearly Normal Galaxies, ed. S. Faber (New York: Springer-Verlag), p 18

Secker, J., et al. 1995, AJ, 109, 1019

van den Bergh, S. 1975, ARAA, 13, 217 